\title{
AVALIAÇÃO DE CATALISADORES DE NÍQUEL E COBALTO SUPORTADOS EM SBA-15 NA SÍNTESE DO BIODIESEL DE SOJA
}

\author{
R. S. EUARDO ${ }^{1}$, J. C. RODRIGUES ${ }^{1}$, E. G. LIMA $^{1}$ e M. G. F. RODRIGUES ${ }^{1}$ \\ ${ }^{1}$ Universidade Federal de Campina Grande, Unidade Acadêmica de Engenharia Química \\ E-mail para contato: raphael_eduardo17@hotmail.com
}

\begin{abstract}
RESUMO - A produção de biodiesel no País se torna cada vez mais efetiva e a utilização de catalisadores heterogêneos na síntese beneficia a pureza dos produtos. Catalisadores a base de níquel e cobalto suportados em SBA-15 na proporção de 5\% de metal foram avaliados na transesterificação do óleo de soja conduzida em reator batelada a $600 \mathrm{RPM}, 200{ }^{\circ} \mathrm{C}$, durante 4 horas. A deposição do níquel sobre suporte foi realizada por dispersão física e o cobalto por impregnação úmida. As amostras foram caracterizadas por DRX, EDX e BET, sendo verificados teores de sílica e metais pré-definidos, formação da estrutura mesoporosa hexagonal, e área específica característica da SBA-15. O biodiesel foi caracterizado em relação à viscosidade cinemática, e conversão a ésteres por cromatografia gasosa. Os resultados de viscosidade $\left(5,69 \mathrm{~mm}^{2} / \mathrm{s} \mathrm{com}\right.$ níquel e $5,35 \mathrm{~mm}^{2} / \mathrm{s}$ com Cobalto) estão dentro dos padrões especificados pela ANP e a conversão a ésteres foi mais eficiente utilizando o catalisador de Cobalto (85\%) quando comparado com o catalisador de níquel $(82 \%)$.
\end{abstract}

\section{INTRODUÇÃO}

Com a dependência mais evidente das energias fósseis, o biodiesel surge como uma possível solução viável, quando respeitados os princípios básicos de sustentabilidade. A implementação deste biocombustível pode representar um avanço inadiável na autonomia energética de diferentes países, reduzindo a poluição atmosférica e a emissão de gases de efeito de estufa, promovendo por outro lado o desenvolvimento agrícola e o tratamento de resíduos, que consequentemente proporcionará a existência de novos postos de trabalho, desencadeando manifestações inequivocamente positivas em termos econômico-sociais (Vultos, 2012).

O biodiesel é um combustível líquido que se obtêm a partir de fontes totalmente renováveis, especialmente de origem vegetal ou animal, como óleos e gorduras. Este biocombustível é biodegradável, não tóxico e é essencialmente livre de compostos sulfurados e aromáticos. O biodiesel puro ou misturado com diesel convencional em certas proporções pode ser usado em motores a diesel, sem qualquer modificação (Sampaio, 2010) porque suas propriedades são semelhantes aos do diesel derivado do petróleo; o biodiesel possui viscosidade adequada, alto número de cetano, características que favorecem a sua tulização no motor sem nenhuma modificação (Noiroj et al., 2009).

A transesterificação, também chamada de alcoólise, é a cisão por intermédio de um álcool onde ocorre a substituição do radical de um éster por um radical de um álcool e pode ser catalisada por ácido ou álcali. Alcoóis adequados incluem o metanol e o etanol entre outros, e o uso adequado de cada um deles depende do custo e dos impactos ambientais (Clark et al., 1984). A transesterificação tem se apresentado como a melhor opção na obtenção do biodiesel, visto que o processo é relativamente simples; aliado a essa técnica, pesquisadores procuram baratear o processo de fabricação do biodiesel utilizando catalisadores reaproveitáveis, desenvolvendo estudos sobre catalisadores heterogêneos que sejam reutilizados na fabricação desse combustível. A rota metílica vem sendo amplamente utilizada, pois o metanol apresenta maior reatividade que o etanol, o que implica em menor tempo e temperatura de reação (Leung et al., 2010). 
Para minimizar os problemas associados ao uso de catalisadores homogêneos, são realizados estudos que podem diminuir os custos de purificação, principalmente pela eliminação de processos secundários e a ausência de reações paralelas, como as de saponificação (Almerindo,2007). Pesquisas com catalisadores ácidos heterogêneos têm sido desenvolvidas para a reação de transesterificação. Estes catalisadores conseguem transesterificar os triacilglicerídeos e esterificar os ácidos graxos livres, além de sofrerem pouca influência da água que por ventura possa estar presente na matéria prima.jhTais catalisadores usam, essencialmente, sólidos com características básicas ou ácidas (Bail, 2007).

Nesse contexto, materiais mesoporosos do tipo SBA-15, compostos essencialmente de sílica, apresentam várias propriedades que os tornam potenciais catalisadores ácidos, destacando suas elevadas áreas superficiais. Além disto, estes materiais possuem paredes dos poros espessas, resultando em uma maior estabilidade hidrotérmica. Entretanto, para torna-los cataliticamente ativos, por suas estruturas serem formadas basicamente de sílica, é necessária a introdução de metais, tais como cobalto, níquel, dentre outros, em suas estruturas (Zhao et al., 1998). Os fatores considerados importantes na dispersão da fase ativa sobre o suporte são os estados de oxidação dos íons metálicos e o caráter do raio atômico, sendo esses relacionados à seletividade e atividade dos catalisadores. A vasta aplicabilidade dos metais do grupo VIII como catalisadores se dá pelo fato destes possuírem estado de oxidação variável e alta atividade para a quebra de ligações $\mathrm{C} \square \mathrm{C}$ (Davda et al., 2004).

Diante da necessidade de novas rotas de produção de peneiras moleculares, a cinza da casca de arroz, produto de resíduos sólidos da indústria de geração de energia adequados à reciclagem, mostra-se promissora como subsídio à síntese de materiais mesoporosos. Uma vez que o TEOS é uma fonte de sílica muito cara, a sua substituição por uma fonte de sílica mais barata se torna atrativa à produção de matérias mesoporosos aplicados na catálise. Devido ao alto teor de sílica presente na cinza, o resíduo passa a ser valorizado. A sílica na casca de arroz é amorfa e transforma-se em sílica cristalina quando aquecida a altas temperaturas (Real et al., 1996).

Este trabalho tem como objetivo avaliar o desempenho dos catalisadores 5\%Co/SBA-15, sintetizado com tetraortosilicato, e $5 \% \mathrm{Ni} / \mathrm{SBA}-15$, sintetizado com cinzas da casca de arroz, na reação de transesterificação do óleo de soja utilizando álcool metílico, observando a influência da fonte de sílica na síntese da peneira molecular SBA-15.

\section{MATERIAS E MÉTODOS}

\subsection{Síntese da Peneira molecular SBA-15}

A peneira molecular do tipo SBA-15 foi sintetizada utilizando o direcionador copolímero tribloco Pluronic P123 (polióxido de etileno - polióxido de propileno - polióxido de etileno, $\mathrm{PEO}_{20} \mathrm{PPO}_{70} \mathrm{PEO}_{20}$ ), ácido clorídrico 37\%, água destilada, e fonte de sílica, empregando o método hidrotérmico proposto por Zhao et al. (1998). Estes reagentes foram misturados para obter um hidrogel reativo de composição molar 1.0 Fonte de Sílica: 0,017 P123: 5,7 HCl: $193 \mathrm{H}_{2} \mathrm{O}$. O direcionador P123 foi dissolvido em água destilada e ácido clorídrico, com agitação e na temperatura de $35^{\circ} \mathrm{C}$. Após homogeneização, adicionou-se a fonte de sílica. Para a síntese da peneira molecular utilizada na preparação do catalisador de níquel suportado, foram utilizadas cinzas da casca de arroz como fonte de sílica, e para a peneira molecular utilizada na preparação do catalisador de cobalto suportado, utilizou-se a fonte de sílica tetraortosilicato (TEOS). A mistura foi mantida sob agitação, na temperatura de $35^{\circ} \mathrm{C}$ por 24 horas $(\mathrm{pH}=1)$, sendo obtido um gel homogêneo, o qual foi transferido para cadinhos de teflon, acondicionados em autoclaves de aço inox. As autoclaves foram postas em estufa previamente aquecida a $100^{\circ} \mathrm{C}$, permanecendo nesta temperatura por 48 horas, seguindo metodologia proposta por Rodrigues (2012). O material obtido foi calcinado em forno mufla sob taxa de aquecimento de $10^{\circ} \mathrm{C} / \mathrm{min}$, até $550^{\circ} \mathrm{C}$, permanecendo nesta temperatura por 4 horas.

As amostras foram denominadas de SBA-15TEOS para a peneira molecular sintetizada com 


\section{9 a 22 de outubro de 2014 \\ Florianópolis/SC}

Tetraortosilicato como fonte de sílica e SBA-15CCA para a peneira molecular SBA-15CCA para a peneira molecular SBA-15 sintetizada com cinzas de casca de arroz como fonte de sílica.

\subsection{Preparação do catalisador 5\% Ni/SBA-15CCA}

O suporte SBA-15 calcinado foi impregnado com concentração de 7,5\% de óxido (em massa), empregando-se a técnica de dispersão física descrita por Xiao, et al. (1998). O óxido de níquel foi obtido pela calcinação do nitrato de níquel hexahidratado $\mathrm{Ni}\left(\mathrm{NO}_{3}\right)_{2} \cdot 6 \mathrm{H}_{2} \mathrm{O}$ a $350^{\circ} \mathrm{C}$ por 4 horas. Previamente a dispersão física, o suporte calcinado e os óxidos foram secos em estufa a $100^{\circ} \mathrm{C}$ por 24 horas. Após esse período, a dispersão física do óxido sobre o suporte foi realizada utilizando almofariz e pistilo. Após 30 minutos de mistura, o material foi calcinado sob fluxo de nitrogênio com vazão de $100 \mathrm{~mL} / \mathrm{g}_{\text {cat }} \cdot \min$, sob taxa de aquecimento de $10^{\circ} \mathrm{C} / \mathrm{min}$, até $550^{\circ} \mathrm{C}$, permanecendo nesta temperatura por 4 horas, com permuta do gás nitrogênio para ar sintético durante esse intervalo.

\subsection{Preparação do catalisador $5 \% \mathrm{Co} / \mathrm{SBA}-15 \mathrm{TEOS}$}

O processo de dispersão do cobalto na peneira molecular SBA-15calcinada foi obtida por impregnação úmida utilizando solução de 0,1 molar de nitrato de cobalto hexahidratado $\mathrm{Co}\left(\mathrm{NO}_{3}\right)_{2} \cdot 6 \mathrm{H}_{2} \mathrm{O}$. Para tal finalidade, $5,0 \mathrm{~g}$ do suporte foi posto em contato com um volume necessário da solução de $\left(\mathrm{Co}\left(\mathrm{NO}_{3}\right)_{2} \cdot 6 \mathrm{H}_{2} \mathrm{O}\right)$ com teor de metal empregado (5\%), sob agitação contínua a temperatura ambiente, por 30 minutos, seguindo metodologia descrita por $\mathrm{Yu}$ et al. (2000). $\mathrm{O}$ material obtido foi submetido à secagem em estufa, a $100{ }^{\circ} \mathrm{C}$ durante $24 \mathrm{~h}$. Após este período o sólido foi submetido ao processo de calcinação, sob fluxo de nitrogênio com vazão de 100 $\mathrm{mL} / \mathrm{g}_{\text {cat }} \cdot \mathrm{min}$, temperatura ambiente até $200^{\circ} \mathrm{C}$ com uma rampa de aquecimento de $10^{\circ} \mathrm{C} / \mathrm{min}$ permanecendo nesta temperatura por $1 \mathrm{~h}$. Após este período, o fluxo de nitrogênio foi trocado por ar sintético, sendo a amostra aquecida a $2^{\circ} \mathrm{C} / \mathrm{min}$ de 200 a $450^{\circ} \mathrm{C}$ permanecendo nesta temperatura por $2 \mathrm{~h}$.

\subsection{Síntese do Biodiesel}

O catalisador $5 \% \mathrm{Ni} / \mathrm{SBA}-15$ foi submetido ao teste reacional de transesterificação do óleo de soja em um sistema composto por um reator batelada de politetrafluoretileno encamisado por uma peça de aço inox nas condições de agitação de 600 RPM, temperatura de $200{ }^{\circ} \mathrm{C}$, tempo de 4 horas, razão molar óleo/álcool de 1/12, e $5 \%$ de catalisador referente à massa de óleo utilizada. $\mathrm{O}$ mesmo sistema e mesmas condições foram utilizados no teste com o catalisador $5 \% \mathrm{Co} / \mathrm{SBA}-15$.

\subsection{Caracterização}

\subsubsection{Difração de Raios X (DRX)}

Os dados coletados das amostras foram analisados utilizando o método do pó empregando-se um difratômetro Shimadzu XRD-6000 com radiação $\mathrm{CuK \alpha}$, tensão de $40 \mathrm{KV}$, corrente de $30 \mathrm{~mA}$, tamanho do passo de $0,0202 \theta$ e tempo por passo de $1,000 \mathrm{~s}$, com velocidade de varredura de $2^{\circ}(2 \theta) / \mathrm{min}$, com ângulo $2 \theta$ percorrido de $0,5^{\circ}$ a $8^{\circ}$ e 35 a $75^{\circ}$.

\subsubsection{Análise Química através do Espectrômetro de Raios X por Energia Dispersiva (EDX)}

As composições das amostras foram analisadas em um espectrômetro EDX-700 Shimadzu.

\subsubsection{Adsorção Física de Nitrogênio (Método de BET)}


$\mathrm{Na}$ obtenção das isotermas de adsorção e dessorção de nitrogênio a $-196^{\circ} \mathrm{C}$ utilizou-se um aparelho ASAP 2020 da Micromeritics. Para isso $100 \mathrm{mg}$ de cada amostra foi previamente tratada nas as seguintes condições: Fase de vacou: Taxa de aquecimento: $10^{\circ} \mathrm{C} / \mathrm{min}$, Temperatura: $90^{\circ} \mathrm{C}$, Vácuo setpoint: $10 \mathrm{micronHg}$, Tempo de vácuo: $60 \mathrm{~min}$. Fase de aquecimento: Taxa de aquecimento: $10^{\circ} \mathrm{C} / \mathrm{min}$ e Temperatura de $350^{\circ} \mathrm{C}$.

\subsubsection{Viscosidade Cinemática}

A viscosidade da amostra foi analisada utilizando o viscosímetro de Cannon Fenske, este equipamento utiliza de um banho a $40^{\circ} \mathrm{C}$ com um tubo capilar de numeração 300 , a determinação da viscosidade é resultado do escoamento total do líquido presente no bulbo do tubo no capilar, multiplicado pelo tempo gasto para esse escoamento, conforme a Equação 1:

$$
V=k t
$$

onde $\mathrm{k}=0,2407 \mathrm{~mm}^{2} / \mathrm{s}^{2}$ é a constante inerente às dimensões do viscosímetro utilizado e $\mathrm{t}(\mathrm{s})$ é o tempo de escoamento do liquido pelo capilar.

\subsubsection{Teor de Éster}

As análises dos ésteres metílicos foram determinadas por cromatografia em fase Gasosa, utilizando o cromatográfo a gás Varian450c com detector de ionização de chamas, coluna capilar de fase estacionária Varian Ultimetal "Select Biodiesel Glycerides + RG" $(15 \mathrm{~m}$ x 0,32mm x $0,45 \mu \mathrm{m})$. A análise consistiu na diluição de $50 \mathrm{mg}$ das amostras em $50 \mathrm{~mL}$ de n-hexano padrão UV/HPLC e posterior injeção de $1 \mu \mathrm{L}$ da solução na coluna capilar. O padrão utilizado foi o padrão interno fornecido pela Varian Inc.

\section{RESULTADOS E DISCUSSÕES}

Os valores dos parâmetros mesoporosos $\mathrm{a}_{0} \mathrm{e}$ dos ângulos $2 \theta$ com suas respectivas distâncias interplanares no plano (hkl) para as amostras se encontram na Tabela 1.

Tabela 1 - Valores dos ângulos $2 \theta$, suas respectivas distâncias interplanares e o parâmetro mesoporoso $\mathrm{a}_{0}$ para o plano $\left(\begin{array}{lll}1 & 0 & 0\end{array}\right)$ para as amostras de SBA-15 e dos catalisadores.

\begin{tabular}{|c|c|c|c|c|}
\hline Amostra & $2 \theta$ & hkl & $\mathrm{d}_{(\mathrm{hkl})}(\mathbf{A})$ & $\mathrm{a}_{0}(\mathrm{~nm})$ \\
\hline SBA-15TEOS & 0,84 & 100 & 104,93 & 12,1 \\
\hline SBA-15CCA & 0,84 & 100 & 103,78 & 11,8 \\
\hline SBA-15TEOS calcinada & 0,94 & 100 & 93,980 & 10,8 \\
\hline SBA-15CCA calcinada & 0,94 & 100 & 92,182 & 10,5 \\
\hline $5 \% \mathrm{Co} / \mathrm{SBA}-15 \mathrm{TEOS}$ & 0,85 & 100 & 102,80 & 11,9 \\
\hline $5 \% \mathrm{Ni} / \mathrm{SBA}-15 \mathrm{CCA}$ & 0,86 & 100 & 101,80 & 11,7 \\
\hline
\end{tabular}

Com a retirada do direcionador P123 pelo processo de calcinação, há um aumento no ordenamento do sistema poroso, aumentando a intensidade dos picos, e uma contração do parâmetro de rede hexagonal, deslocando o ângulo dos picos para valores maiores (Yu et al., 2000). Constata-se pelos valores (Tabela 1), a contração do parâmetro de rede hexagonal após a calcinação: SBA-15TEOS diminui de 12,1 para 10,8; SBA15CCA reduz de 11,8 para 10,5. Na Figura 1 estão apresentados os resultados de difração de raios X das 
peneiras moleculares SBA-15 sintetizadas com TEOS e Cinzas da casca de arroz antes e após calcinação, como também os padrões obtidos para os catalisadores 5\%Co/SBA-15 e 5\%Ni/SBA-15.
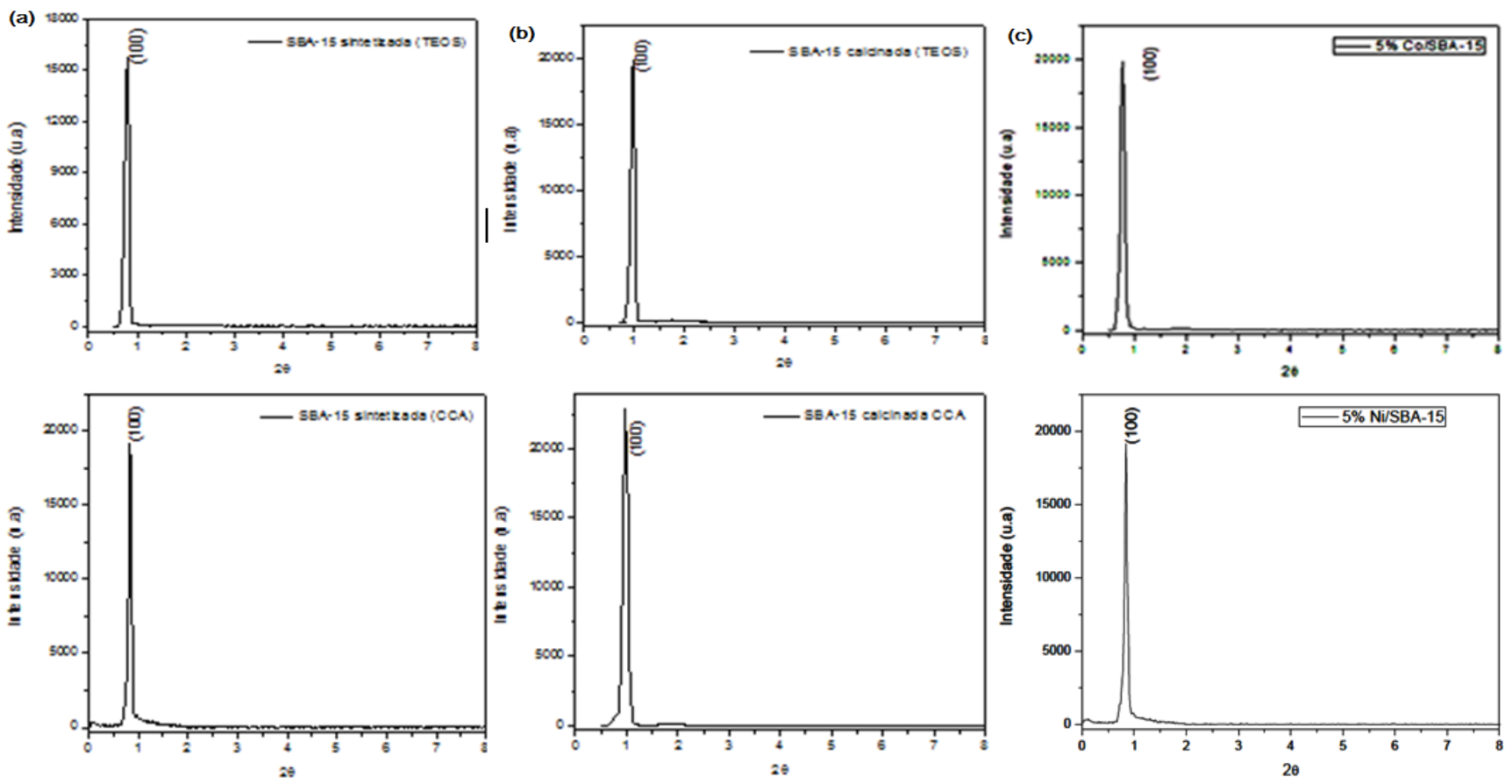

Figura 1 - Padrões de difração de raios X: (a) SBA-15TEOS, (b) SBA-15CCA, (c) SBA-15 TEOS calcinada, (d) SBA-15CCA calcinada, (e) catalisador 5\% Co/SBA-15TEOS, (f) catalisador 5\%Ni/SBA-15CCA.

A estrutura hexagonal ordenada da peneira molecular mesoporosa SBA-15 foi verificada nas amostras (a) e (b) e está de acordo com a literatura (Zhao et al. 1998; Rodrigues, 2011), onde pode ser visto a presença do pico principal de difração, característico desse material, referentes ao plano cristalino, cujo índice de Miller é (100). Após calcinação, amostras (c) e (d), e impregnação dos metais, amostras (e) e (f) a estrutura hexagonal foi mantida indicando que não houve mudanças significativas nos mesoporos da peneira molecular SBA-15.

$\mathrm{Na}$ Tabela 2 são apresentados os resultados da análise química das amostras de SBA-15 e dos catalisadores obtidos.

Tabela 2 - Composição química das amostras de SBA-15 e do catalisador.

\begin{tabular}{c|c|c|c|c}
\hline Amostra & $\mathrm{SiO}_{2}(\%)$ & $\mathrm{Co}_{3} \mathrm{O}_{4}(\%)$ & $\mathrm{NiO}(\%)$ & Impurezas (\%) \\
\hline SBA-15TEOS & 99,5 & & & 0,5 \\
\hline SBA-15TEOS Calcinada & 99,6 & & & 0,4 \\
\hline SBA-15CCA & 99,3 & - & - & 0,7 \\
\hline SBA-15CCA Calcinada & 99,5 & - & - & 0,5 \\
\hline 5\%Co/SBA-15TEOS & 92,3 & 7,0 & - & 0,7 \\
\hline 5\%Ni/SBA-15CCA & 92,7 & - & 6,8 & 0,5 \\
\hline
\end{tabular}

De acordo com os resultados de composição química, as amostras de SBA-15 apresentaram alto teor de sílica $\left(\mathrm{SiO}_{2}\right)$, previsto para este tipo de material (Zhao et al., 1998). O catalisador 5\%Co/SBA-15 apresentou teor de óxido de cobalto de $7,01 \%$. Na forma de elemento o valor obtido foi de $5,02 \%$. Para o catalisador $5 \% \mathrm{Ni} / \mathrm{SBA}-15$, o teor de óxido de níquel observado foi de de $6,8 \%$ e na forma de metal o valor obtido foi de 
5,3\%. Estes valores ficaram próximos do valor pretendido de 5\%, indicando eficiência no processo incorporação dos metais na estrutura da peneira molecular SBA-15.

$\mathrm{Na}$ Figura 2 estão apresentadas as isotermas de adsorção-dessorção de $\mathrm{N}_{2}$ a $-196{ }^{\circ} \mathrm{C}$ referentes às amostras de SBA-15(TEOS) e SBA-15(Cinzas da casca de arroz), e do catalisadores 5\%Co/SBA-15 e $5 \% \mathrm{Ni} / \mathrm{SBA}-15$.
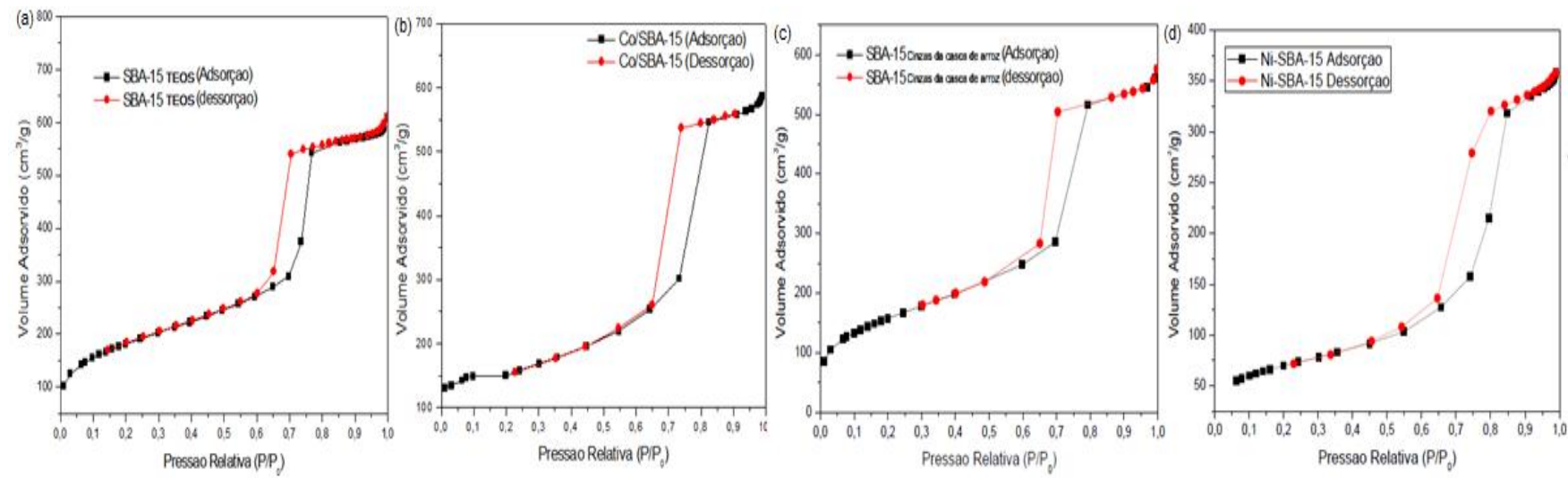

Figura 4 - Isotermas de adsorção-dessorção de $\mathrm{N}_{2}$ a $-196^{\circ} \mathrm{C}$ : (a) SBA-15(TEOS), (b) 5\%Co/SBA15, (c) SBA15(Cinzas da casca de arroz) e (d) 5\% Ni/SBBA-15.

Verificou-se por meio das análises de adsorção-dessorção de $\mathrm{N}_{2}$ isotermas do tipo IV e histerese do tipo $\mathrm{H} 1$, constituída por dois ramos verticais durante um intervalo de 0,6 a $0,8\left(\mathrm{P} / \mathrm{P}_{0}\right)$ no eixo das abscissas, características de materiais mesoporosos do qual a SBA-15 faz parte. Na tabela 3 são apresentados os resultados das propriedades texturais da SBA-15 e dos catalisadores.

Tabela 3 - Propriedades texturais da SBA-15 e dos catalisadores obtidos.

\begin{tabular}{c|c|c|c}
\hline Amostra & $\mathrm{S}_{\mathrm{BET}}\left(\mathrm{m}^{2} / \mathrm{g}\right)$ & $\mathrm{VP}_{\text {total }}\left(\mathrm{cm}^{3} / \mathrm{g}\right)$ & $\mathrm{e}(\mathrm{nm})$ \\
\hline SBA-15 (TEOS) & 763 & 0,98 & 8 \\
\hline SBA-15 (Cinzas da casca de arroz) & 627 & 0,96 & 8 \\
\hline 5\%Co/SBA-15 & 471 & 0,77 & - \\
\hline 5\%Ni/SBA-15 & 246 & 0,54 & - \\
\hline
\end{tabular}

O valor da área superficial específica da SBA-15CCA de $627 \mathrm{~m}^{2} / \mathrm{g}$ foi inferior ao valor encontrado para SBA-15TEOS de $763 \mathrm{~m}^{2} / \mathrm{g}$, como também os respecitivos volume de poro, que apresentaram valores de $0,77 \mathrm{~cm}^{3} / \mathrm{g}$ e $0,54 \mathrm{~cm}^{3} / \mathrm{g}$. Porém a SBA-15CCA apresentou espessura de parede semelhante ao da peneira molecular SBA-15TEOS. Os catalisadores, em ambos os casos, a área e o volume total diminuíram, significativamente. Ao comparar o catalisador 5\%Co/SBA-15TEOS com o suporte SBA-15TEOS, constata-se uma redução de $292 \mathrm{~m}^{2} / \mathrm{g}$. A redução da área específica de $375 \mathrm{~m}^{2} / \mathrm{g}$ foi mais acentuada no catalisador $5 \% \mathrm{Ni} / \mathrm{SBA}-15$ quando comparado com o suporte SBA-15CCA. Isto pode ter sido causado pelo bloqueio dos poros da peneira molecular SBA-15 pela aglomeração dos óxidos e/ou colapso parcial da estrutura mesoporosa (Girardon et al., 2005). A aglomeração por óxidos é a hipótese mais aceita, visto que a estrutura da peneira molecular se manteve após imprengnação dos metais.

Os resultados dos testes de viscosidade cinemática e conversão dos triacilglicerídeos do óleo de soja à ésteres, utilizando os catalisadores 5\% Co/SBA-15TEOS e 5\%Ni/SBA-15CCA, se encontram na Tabela 4. 
Tabela 4 - Viscosidade cinemática e conversão a ésteres do óleo de soja.

\begin{tabular}{c|c|c}
\hline Catalisador & Viscosidade $\left(\mathrm{mm}^{2} / \mathrm{s}\right)$ & Conversão $(\%)$ \\
\hline $5 \% \mathrm{Co} / \mathrm{SBA}-15$ & 5,35 & 85 \\
\hline $5 \% \mathrm{Ni} / \mathrm{SBA}-15$ & 5,69 & 82 \\
\hline
\end{tabular}

Os valores de viscosidade obtidos com os catalisadores 5\%Co/SBA-15 e 5\% Ni/SBA-15 pela reação de transesterificação do óleo de soja estão dentro dos limites aceitos pela ANP. A viscosidade apresenta-se como uma das principais propriedades em virtude da influência direta na qualidade do combustível. A análise por cromatografia gasosa forneceu resultado satisfatório q uanto à porcentagem de ésteres totais. Pode-se afirmar que houve conversão elevada e alto rendimento em ésteres.

\section{CONCLUSÕES}

Neste trabalho, cinzas da casca de arroz amorfa foram utilizadas com fonte de sílica para síntese da peneira molecular SBA-15. Os resultados obtidos mostraram que foi possível sintetizar a peneira molecular SBA-15 utilizando a cinza da casca de arroz como fonte de sílica e que suas características se aproximam às da peneira molecular SBA-15 utilizando fontes convencionais.

A adição dos metais, coalto e níquel, na peneira molecular SBA-15 não causou modificação em sua estrutura hexagonal, porém causou diminuição significativa na área específica superficial, sendo mais acentuada essa redução no catalisador $5 \% \mathrm{Ni} / \mathrm{SBA}-15$.

A transesterificação do óleo de soja com metanol apresentou conversão de $82 \%$ utilizando o catalisador $5 \% \mathrm{Ni} / \mathrm{SBA}-15$, resultado menor que a conversão de $85 \%$ obtida com o catalisador 5\% Co/SBA-15. A diminuição da área específica poder ter influência na diferença entre os resultados. Pelas análises de viscosidade cinemática, foi verificado que o biodiesel obtido com óleo de soja apresentou resultados semelhantes às do diesel e se adéquam aos limites estabelecidos pela ANP. Se tratando de um material heterogêneo, a utilização dos catalisadores \%Co/SBA-15 e 5\%Ni/SBA-15 proporciona grande perspectiva na reação de transesterificação por apresentar rendimento satisfatório e otimização dos processos secundários de separação.

\section{REFERÊNCIAS}

ALMERINDO, G. I. Catalisador Heterogêneo $\mathrm{MgO}$ obtido por diferentes métodos para a produção de biodiesel - Rota Etílica. In: CONGRESSO BRASILEIRO DA REDE BRASILEIRA DE TECNOLOGIA DE BIODIESEL, 2007, Brasília. II Congresso Brasileiro da Rede Brasileira de Tecnologia de Biodiesel, 2007.

BAIL, A. Uso de diferentes sólidos inorgânicos como catalisadores heterogêneos de reações de transesterificação. In: CONGRESSO DA REDE BRASILEIRA DE TECNOLOGIA DE BIODIESEL, v. 2, 2007, Brasília, DF: Resumos do II Congresso da Rede Brasileira de Tecnologia de Biodiesel. Brasília, DF: ABIPTI, v.1, 2007

CLARK, S. J.; WANGNER, L.; STROCK, M.D.; PIENNAAR, P. G. Methyl and ethyl soybean esters as renewable fuels for diesel engines. Journal of the American Oil Chemists' Society, v.61, p.1632-1638,1984.

DAVDA, R. R.; SHABAKER, J.W.; HUBER, G.W. A review of catalytic issues and process conditions for renewable hydrogen and alkanes by aqueous-phase reforming of oxygenated hydrocarbons over supported metal catalysts. Applied Catalysis. v.56, p.171- 186, 2004. 
GIRARDON, J. S.; LERMONTOV, A. S.; GENGEMBRE, L.; CHERNAVSKII, P. A. C; GRIBOVALCONSTANT, A.; KHODAKOV, A. Y. Effect of cobalt precursor and pretreatment conditions on the structure and catalytic performance of cobalt silica-supported Fischer-Tropsch catalysts. Journal of Catalysis, v.230, p.339-352, 2005.

LEUNG, D. Y. C.; WY, X.; LEUNG, M. K. H. A review on biodiesel production using catalyzed transesterification. Applied Energy. v.87, p.108-1095, 2010.

NOIROJ, K.; INTARAPONG, P.; LUENGNARUEMITCHAI, A.; JAI-IN, S. A comparative study of $\mathrm{KOH} / \mathrm{Al}_{2} \mathrm{O}_{3}$ and $\mathrm{KOH} / \mathrm{NaY}$ catalysts for biodiesel production via transesterification from palm oil. Renewable Energy, v.34, p.1145-1150, 2009.

REAL, C; ALCALA, M.; CRIADO, J. Preparation of Silica from Rice Husks. Journal of the American Ceramic Society. v.79, p.2012-2016, 1996.

RODRIGUES, J. J.; PECCHI, G.; FERNANDES, F. A. N.; RODRIGUES, M. G. F. Ruthenium promotion of Co/SBA-15 catalysts for Fischer-Tropsch synthesis in slurry-phase reactors. Journal of Natural Gas Chemistry, Vv.21, p. 722-728, 2012.

SAMPAIO, M. J. F. Produção de Biodiesel por catálise heterogênea. Dissertação (Mestrado) -Instituto Politécnico de Bragança, Escola Superior de Tecnologia e de Gestão. Bragança, p.70, 2008.

VULTOS, D. A. A. N. A utilização do Biodiesel no Concelho do Seixal. Dissertação (mestrado) - Faculdade de Ciências Sociais e Humanas, Universidade Nova de Lisboa, Programa de Pós-Graduação em Ecologia Humana e Problemas Sociais Contemporâneo, Lisboa, p.91, 2014.

YU, J.; SHI, J. L.; WANG, L.Z.; RUAN, M. L.; YAN, D. S. Room temperature synthesis of mesoporous aluminosilicate materials. Ceramics International. v.26, p.359-362, 2000.

ZHAO, D., HUO, Q., FENG, J., B.F. CHMELKA. G.D. Stucky, Nonionic Triblock and Star Diblock Copolymer and Surfactant Syntheses of Highly Ordered, Hydrothermally Stable, Mesoporous Silica Structures. Journal of the American Chemical Society. v.120, p.6024-6036, 1998. 